\title{
Targeting breast cancer stem cells by dendritic cell vaccination in humanized mice with breast tumor: preliminary results
}

This article was published in the following Dove Press journal:

OncoTargets and Therapy

21 July 2016

Number of times this article has been viewed

\author{
Phuc Van Pham' \\ Hanh Thi Le' \\ Binh Thanh $\mathrm{Vu}^{\prime}$ \\ Viet Quoc Pham' \\ Phong Minh Le' \\ Nhan Lu-Chinh Phan' \\ Ngu Van Trinh' \\ Huyen Thi-Lam Nguyen' \\ Sinh Truong Nguyen' \\ Toan Linh Nguyen ${ }^{2}$ \\ Ngoc Kim Phan' \\ 'Laboratory of Stem Cell Research \\ and Application, University of Science, \\ Vietnam National University, Ho Chi \\ Minh City, ${ }^{2}$ Vietnam Military Medical \\ University, Ha Dong, Ha Noi, Vietnam
}

Correspondence: Phuc Van Pham Laboratory of Stem Cell Research and Application, University of Science, Vietnam National University, 227 Nguyen Van Cu, District 5, Ho Chi Minh City, Vietnam

Tel +84903870153

Email pvphuc@hcmuns.edu.vn
Background: Breast cancer (BC) is one of the leading cancers in women. Recent progress has enabled $\mathrm{BC}$ to be cured with high efficiency. However, late detection or metastatic disease often renders the disease untreatable. Additionally, relapse is the main cause of death in $\mathrm{BC}$ patients. Breast cancer stem cells (BCSCs) are considered to cause the development of BC and are thought to be responsible for metastasis and relapse. This study aimed to target BCSCs using dendritic cells (DCs) to treat tumor-bearing humanized mice models.

Materials and methods: NOD/SCID mice were used to produce the humanized mice by transplantation of human hematopoietic stem cells. Human BCSCs were injected into the mammary fat pad to produce BC humanized mice. Both hematopoietic stem cells and DCs were isolated from the human umbilical cord blood, and immature DCs were produced from cultured mononuclear cells. DCs were matured by BCSC-derived antigen incubation for 48 hours. Mature DCs were vaccinated to $\mathrm{BC}$ humanized mice with a dose of $10^{6}$ cells $/$ mice, and the survival percentage was monitored in both treated and untreated groups.

Results: The results showed that DC vaccination could target BCSCs and reduce the tumor size and prolong survival.

Conclusion: These results suggested that targeting BCSCs with DCs is a promising therapy for BC.

Keywords: breast cancer, breast cancer stem cells, targeting cancer therapy, humanized mice, targeting cancer stem cells

\section{Introduction}

Breast cancer (BC) is the second leading cause of cancer-related death in women. ${ }^{1}$ Despite advances in treatment methods, such as surgery, chemotherapy, radiation therapy, and biological therapy, the percentage of death in $\mathrm{BC}$ patients remains high. Although targeted therapies using antibodies, such as pertuzumab and trastuzumab, have significantly improved the treatment of BC in recent years,,$^{2-4}$ some investigations reported that $30 \%-70 \%$ of $\mathrm{BC}$ patients relapse after 5 years. ${ }^{5}$

In recent years, dendritic cell (DC) vaccination has emerged as a promising therapy for cancer treatment. DCs are professional antigen-presenting cells in the human body that originate from bone marrow precursors. ${ }^{6}$ In an immature state, DCs exhibit high endocytic activity and low T-cell activation. Upon contact with an antigen, they become mature and can strongly activate the T-cells via cell-cell contact or by producing a pool of cytokines. ${ }^{7}$ These cells highly express costimulators, major histocompatibility complex molecules (CD80, CD86), and CD40. Through interaction between CD40 (on DCs) and CD40 ligand (on T-cells), DCs can proliferate and present the 
antigens to T-cells. ${ }^{8}$ In BC patients, DCs reportedly exhibit abnormalities that prevent them from efficiently presenting the tumor antigens to T-cells. In fact, it has been shown that DCs in cancer patients exhibit reduced antigen uptake, reduced antigen processing, low expression of costimulators, weak migration, and decreased interleukin-12 (IL-12) production. ${ }^{9}$ It was previously demonstrated that DCs in $\mathrm{BC}$ are dysfunctional and show weaker migration to lymph nodes, lower expression of human leukocyte antigen (HLA) and CD86, and lower ability to induce IL-12 secretion in vitro compared with those in healthy patients..$^{10}$ To address these problems, DC therapy was used to produce a large number of functional DCs ex vivo. Specifically, both hematopoietic stem cells (HSCs) and monocytes were collected and induced to DCs using a cocktail of granulocyte-macrophage colonystimulating factor (GM-CSF) and IL-4. ${ }^{11}$ Subsequently, the immature DCs were loaded with antigens in the form of DNA, RNA, proteins, peptides, or cell lysates to produce the mature DCs for further applications.

DC therapies have been used in both preclinical and clinical trials for various cancers, such as prostate cancer, ${ }^{12,13}$ multiple myeloma, ${ }^{14,15}$ renal cell carcinoma, ${ }^{16,17}$ pancreatic cancer, ${ }^{18,19}$ leukemia, ${ }^{20}$ melanoma, ${ }^{21}$ colorectal cancer, ${ }^{22,23}$ glioma, ${ }^{24,25}$ and BC. ${ }^{26-28}$ In almost all cases, DC vaccination was demonstrated to be a safe and effective method for treating metastatic patients..$^{29}$ Importantly, some DC vaccinations have been approved by governmental regulatory agencies as official methods to treat cancers. For example, Sipuleucel-T has been approved by the United States Food and Drug Administration to treat human prostate cancer, ${ }^{30}$ and Vaccell has been approved by the Japanese Food and Drug Administration. To date, there are $>289$ clinical studies of DC-based cancer vaccines that are registered and under investigation (https:// www.ClinicalTrials.gov). More importantly, among the 289 cases, six are in Phase III, and two are in Phase IV.

Recently, it has been shown that DC vaccination can improve $\mathrm{BC}$ treatment. For example, Brossart et $\mathrm{a}^{31}$ showed that DC vaccination with HLA-A2-restricted HER2 or MUC peptide-pulsed DCs induced immunologic responses in patients. However, the clinical efficacy of DC vaccination was not recorded in this study. In a separate study, Avigan et $\mathrm{a}^{32}$ fused DCs with BC cells and recorded immunological and antitumor responses. More recently, Qi et al ${ }^{33}$ adopted a novel inducing method for DCs by using tumor lysate, but the results were limited, with only a partial response. DC vaccine, in combination with IL-2 ${ }^{28}$ or IL-12, also recorded specific immunity against introduced antigen. In order to improve the outcome, some investigators combined DCs with cytokine killer cells and found significant improvement in the progression-free survival and overall survival of patients. ${ }^{34}$ Thus, to date, DC therapy has had limitations in the improvement of the clinical outcome.

Breast cancer stem cells (BCSCs) were discovered over 10 years ago, by Al-Hajj et al, ${ }^{35}$ and have been shown to be the cause of breast tumor development and the drivers of therapeutic resistance in BC. ${ }^{36,37} \mathrm{New}$ therapies aimed at targeting BCSCs have shown an increase in patient outcome. ${ }^{38,39}$ Therefore, we hypothesized that the existence of BCSCs in tumors may be responsible for the low efficacy of DC therapy. In this study, we aimed to evaluate the preclinical trial efficacy of a DC vaccination from DCs primed from BCSC lysate using BC humanized mice models.

\section{Materials and methods}

\section{Animals, BCSCs, and umbilical cord blood}

NOD/SCID mice were bought from Jackson Laboratory (Charles

River). Mice manipulations were approved by the Institutional Animal Care and Use Committee of Stem Cell Research and Application Laboratory, University of Science, Vietnam National University, Ho Chi Minh. All mice were housed in individual ventilated cages and were carefully monitored daily as The Institutional Animal Care and Use Committee guidelines (followed by Guide for the Care and Use of Laboratory Animals, Eighth Edition, National Institute of Health, US, published by The National Academies Press, Washington, DC, USA).

BCSCs were used from the previously published study. ${ }^{40}$ BCSCs were thawed and allowed to proliferate in suitable conditions. BCSCs were cultured in mammosphere medium without fetal bovine serum supplement (ie, Dulbecco's Modified Eagle's Medium/F12 supplemented with 1\%(v/v) prostate-specific antigen, $2 \%(\mathrm{v} / \mathrm{v}) \mathrm{B}-27$ supplement, $20 \mathrm{ng} / \mathrm{mL}$ epidermal growth factor and basic fibroblast growth factor, $4 \mathrm{ng} / \mathrm{mL}$ heparin, and $10 \mu \mathrm{g} / \mathrm{mL}$ insulin) in $10 \% \mathrm{O}_{2}, 5 \% \mathrm{CO}_{2}$, as published previously. ${ }^{41} \mathrm{BCSCs}$ were validated by flow cytometry using the surface markers with phenotype $\mathrm{CD} 44^{+} \mathrm{CD} 24^{-}$before being used in the experiments. Briefly, BCSCs were stained with both anti-CD44 monoclonal antibody conjugated with antigen-presenting cells and anti-CD24 monoclonal antibody conjugated with fluorescein isothiocyanate (FITC) (BD Biosciences, San Jose, CA, USA). Stained cells were analyzed in FASCalibur machine with CellQuest Pro at 10,000 events.

Umbilical cord blood (UCB) was collected as described previously. ${ }^{42}$ Briefly, UCB was collected from the umbilical cord vein with informed consent from the mother. The collection was performed in accordance with the ethical standards of the local ethics committee (Van Hanh General Hospital, Ho Chi Minh City, Vietnam). 


\section{Isolation of HSCs from UCB}

In this study, HSCs were used as unpurified, mononuclear cells (MNCs) that were isolated from UCB. To isolate MNCs, each UCB unit was diluted into a ratio of 1:1 with phosphatebuffered saline (PBS), and $10 \mathrm{~mL}$ of diluted blood was loaded on to $25 \mathrm{~mL}$ Ficoll Hypaque solution $(1.077 \mathrm{~g} / \mathrm{mL}$; Code 10771; Sigma-Aldrich Co., St Louis, MO, USA) in a $50 \mathrm{~mL}$ tube. After centrifuging at 2,500 rpm for 5 minutes, MNCs were derived from the interphase layer and washed twice with PBS.

To determine the dose of HSCs for transplantation, the obtained MNCs were used to enumerate the HSCs. The number of HSCs was determined using an Enumeration Pro-Count Kit (BD Biosciences) following the manufacturer's guidelines.

\section{Humanized mice}

NOD/SCID mice were intraperitoneally injected with busulfan $(25 \mathrm{mg} / \mathrm{kg}$ ) prepared in dimethyl sulfoxide. After 48 hours, total white blood cells (WBCs) and body weight were measured for each mouse. Only mice with WBCs $<1,000$ cells $/ \mathrm{mL}$ were used to graft HSCs. Mice were anesthetized using ketamine (100 $\mu \mathrm{L} /$ mouse). Mice tails were relaxed in warm water $\left(37^{\circ} \mathrm{C}\right)$ for $3-5$ minutes. Tails were then cleaned with $70 \%$ alcohol. HSCs $\left(1 \times 10^{6}\right)$ in MNCs were transfused into the tail vein with a total volume of $0.5 \mathrm{~mL}$ at a rate of $10 \mathrm{~mL} / \mathrm{h}$ using an electronic pump.

The presence of human cells was determined by measuring the percentage of human leukocytes (CD45 cells) in the peripheral blood. HSC localized in the bone marrow was determined by the existence of human HSCs (CD34 cells) in the bone marrow. HSC differentiation was determined based on the presence of CD3, CD4, CD8, CD1a, and CD56 in the peripheral blood. To determine the presence of CD1a, CD3, CD4, CD8, CD34, CD56, and CD45, peripheral blood was collected at the tail vein with anticoagulation agent (ACD-A; BD Biosciences). Blood samples were stained with antibodies for CD1a, CD3, CD4, CD8, CD34, CD56, and CD45 conjugated with FITC for 20 minutes at room temperature. Then, blood was lysed with Pharm Lyse Lysing buffer (BD Biosciences). Lysed blood was diluted with $100 \mu \mathrm{L}$ sheath fluid for analysis in a FACSCalibur machine. The human immunoglobulin $\mathrm{G}(\mathrm{IgG})$ was detected and measured in peripheral blood using enzyme-linked immunosorbent assay (ELISA) technique.

\section{BC humanized mice}

Humanized mice were injected with $1 \times 10^{6} \mathrm{BCSCs}$ with GFPs into the fat pad to produce $\mathrm{BC}$ humanized mice.
Mice were monitored for tumor growth and invasion. BCSCs were transduced with GFP as described in a previously published study. ${ }^{43}$

\section{Immature DC production}

MNCs were cultured in RPMI 1640 supplemented with 10\% fetal bovine serum, $20 \mathrm{ng} / \mathrm{mL}$ GM-CSF, $50 \mathrm{ng} / \mathrm{mL}$ IL-4, 1\% L-glutamine, and 1\% penicillin/streptomycin (Sigma-Aldrich Co.). At day 3 of culturing, $75 \%$ of the medium was removed, and fresh medium was added. On day 6, DC maturation was induced by supplementing the complete medium with $10,000 \mathrm{ng} / \mathrm{mL}$ BCSC-derived lysate and tumor necrosis factor- $\alpha(100 \mathrm{ng} / \mathrm{mL})$. At day 3, cells were harvested and used in subsequent experiments.

To evaluate the phenotype of mature DCs, the differentiated cells were stained with specific surface markers CD40 (CD40-FITC), CD80 (CD80-PERCP), CD86 (CD86-PE), and HLA-DR (HLA-DR-FITC) in staining buffer (PBS pH 7.4, $0.5 \%$ bovine serum albumin, $0.02 \%$ azide). The stained cells were analyzed by BD FACSCalibur flow cytometer.

T-lymphocyte proliferation stimulated by DCs and measurement of IL-12 were evaluated as described in a previously published study. ${ }^{44}$ There were five experimental groups with different ratios of DCs:lymphocytes $(0.25: 100$, $0.5: 100,1: 100,2: 100$, and 8:100) and three control groups with DCs + phytohemagglutinin (PHA), PHA alone, or PHA + lymphocytes. The T-lymphocyte concentration was measured by 3-(4,5-dimethylthiazol-2-yl)-2,5-diphenyltetrazolium bromide assay kit according to the manufacturer's instructions (Sigma-Aldrich Co.). Optical density values were read at a wavelength of $490 \mathrm{~nm}$ with the reference wavelength of $620 \mathrm{~nm}$. The stimulation ability of DCs was calculated based on $A$-values. $A$-values were offset from optical density values measured for control samples (lymphocyte + PHA) and experimental groups. To measure IL-12 concentration, mature DCs were incubated in the fresh culture medium in a $24-w e l l$ plate for 24 hours. Then, supernatants were collected and frozen at $-80^{\circ} \mathrm{C}$ until analysis. IL-12 concentration in the supernatants was measured by ELISA kits (IL-12 High Sensitivity Human ELISA Kit; Abcam, Cambridge, UK), and the results were analyzed with the DTX880 Multimode Detector (Beckman Coulter, Inc., Brea, CA, USA).

\section{BCSC antigen production}

BCSCs grown to $70 \%-80 \%$ confluence were detached with $0.25 \%$ trypsin/ethylenediaminetetraacetic acid and washed two times with Dulbecco's PBS. BCSC lysates were generated from $5 \times 10^{6}$ tumor cells by three rapid freeze-thaw 
cycles ( 7 minutes in liquid nitrogen and 7 minutes in the water bath). The tumor cell lysates were then spun at $700 \mathrm{rpm}$ at $4^{\circ} \mathrm{C}$ for 10 minutes to remove cellular debris, and the supernatant was collected. All lysates were stored at $-80^{\circ} \mathrm{C}$ until use.

\section{DC vaccination and efficiency monitoring plan}

On day 3, tumor-bearing mice were treated with intravenous injections of mature DCs in $100 \mu \mathrm{L}$ of PBS. Tumor-bearing mice were sorted into two groups: 1) the treated group (ten mice), which was injected with $1 \times 10^{6}$ primed mature DCs (only one time), and 2) the untreated control (ten mice), which was injected with $100 \mu \mathrm{L}$ of PBS.

From day 0 until day 30 of treatment, tumor size was measured using calipers in two dimensions, and size was calculated using the following formula: $a \times b^{2} / 2$, where " $a$ " is the tumor length and " $b$ " is the diameter. ${ }^{45}$ The survival percentages of the mice were recorded in both treated and untreated groups during the 120 days of treatment.

\section{Statistical methods}

The results were expressed as the mean $\pm \mathrm{SD}$. One-way analysis of variance and two-tailed tests were utilized for all statistical analyses performed with GraphPad Prism software, Version 4.0 (GraphPad Software, Inc., La Jolla, CA, USA). $P$-values $<0.05$ were considered statistically significant.

\section{Results}

\section{Humanized mice models}

In order to generate humanized mice, we treated NOD/SCID mice with $25 \mathrm{mg} / \mathrm{kg}$ busulfan in order to destroy the murine bone marrow. WBCs strongly decreased from $1,788 \pm 665$ cells $/ \mu \mathrm{L}$ to $763 \pm 571$ cells $/ \mu \mathrm{L}$, while in control mice, WBCs did not significantly change $(1,659 \pm 571$ cells $/ \mu \mathrm{L}$ to $1,518 \pm 207$ cells/ $\mu \mathrm{L})$. Busulfan also strongly decreased CD4 cells from $11.26 \% \pm 2.45 \%$ to $1.83 \% \pm 0.70 \%$. However, the percentage of CD8 cells nonsignificantly reduced compared with that before busulfan injection.

Ten NOD/SCID mice in the experimental group were injected with HSC via the tail vein, and ten NOD/SCID mice in the control group were injected with PBS. HSCs were counted based on the expression of $\mathrm{CD} 45^{+} \mathrm{CD} 34^{+}$and colony-forming unit assay (Figure 1).

HSC transplantation caused many biological effects on mice, including an increase in body weight, changes in WBCs, existence of human leukocytes in murine peripheral blood, HSC homing in murine bone marrow, and multiple lineage differentiation of human HSCs in mice. Importantly, the HSC transplantation extended the survival of transplanted mice.

The results showed that experimental mice significantly increased their body weight, compared with that of control mice, especially in the first 3 weeks (the body weight increased from $23.18 \pm 0.15 \mathrm{~g}$ to $24.22 \pm 0.32 \mathrm{~g}$ in the first week and increased again to $24.68 \pm 0.27 \mathrm{~g}$ in the second week after HSC transplantation). However, after 4 weeks of transplantation, the body weight suddenly decreased from $24.14 \pm 0.48 \mathrm{~g}$ to $21.94 \pm 1.6 \mathrm{~g}$. The WBCs in experimental mice significantly decreased from the first week to the second week $(3,504 \pm 420$ cells $/ \mu \mathrm{L}$ to $975 \pm 218$ cells $/ \mu \mathrm{L})$. However, after the second week, the WBCs gradually increased and reached $1,947 \pm 566$ cells $/ \mu \mathrm{L}$ but then gradually decreased to $825 \pm 188$ cells $/ \mu \mathrm{L}$ at the fourth week.

By investigating the existence of human leukocytes (CD45 cells) (Figure 2), the results showed that after 2 weeks, the human leukocytes slightly increased, but after that this percentage rapidly increased up to the fourth week. However, the human CD45 cell percentage changed between the ten mice with large amplitude. The highest percentage of human CD45 leukocyte in peripheral blood achieved 37\% in WBCs isolated from peripheral blood at day 28 after HSC transplantation.

After 28 days of transplantation, transplanted mice were used to evaluate the existence of CD34 cells in bone marrow (Figure 2C and D). After staining with anti-human CD34FITC, the results showed that $100 \%$ of mice had HSC homing to the bone marrow. Compared with the total of HSCs in bone marrow, the results showed that the highest human HSCs in bone marrow achieved $38.58 \%(n=3)$. In addition, the existence of different kinds of human leukocytes was investigated, using CD3, CD4, CD8, CD19, CD45, and CD56 as markers (Figure 2E-J). The results showed that after 35 days of transplantation, the leukocytes appeared in the peripheral blood, and the percentages of which rapidly increased in 42 days, 49 days, and 55 days.

The function of human B-cells was also evaluated using human IgG in peripheral blood. The human IgG concentrations at 42 days, 49 days, and 55 days were $78 \pm 9.6 \mu \mathrm{g} / \mathrm{mL}$, $89 \pm 13.1 \mu \mathrm{g} / \mathrm{mL}$, and $110 \pm 15.6 \mu \mathrm{g} / \mathrm{mL}$, respectively. However, the HSC transplantation significantly reduced survival in mice. Compared with the control group, after 120 days, there were $20 \%$ of mice alive, while only $6.67 \%$ of mice alive in the experimental group. HSC transplantation also caused mice to die earlier than in the control group. In fact, 75 days after transplantation, only $\sim 26.67 \%$ of HSC-transplanted 

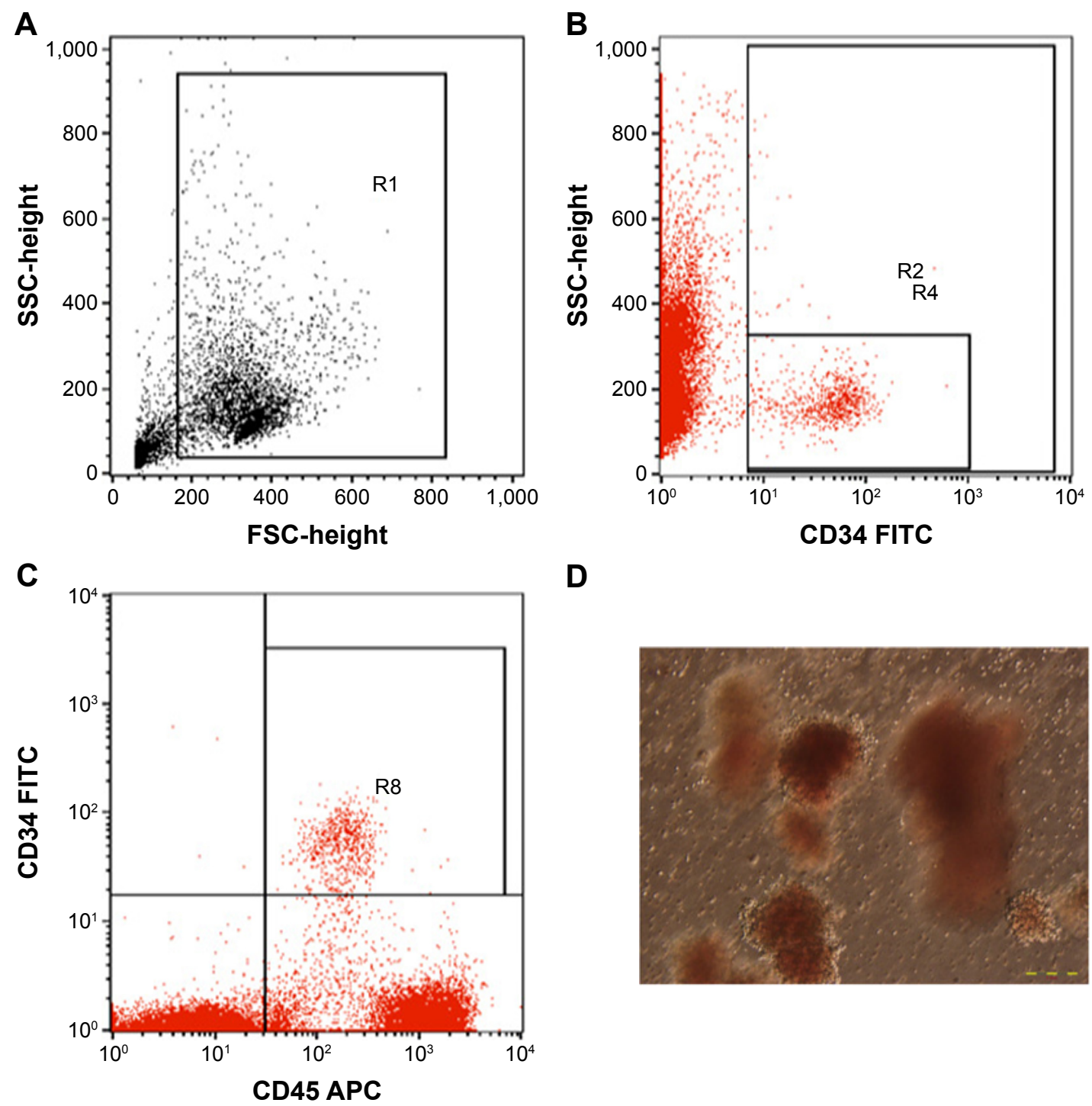

D

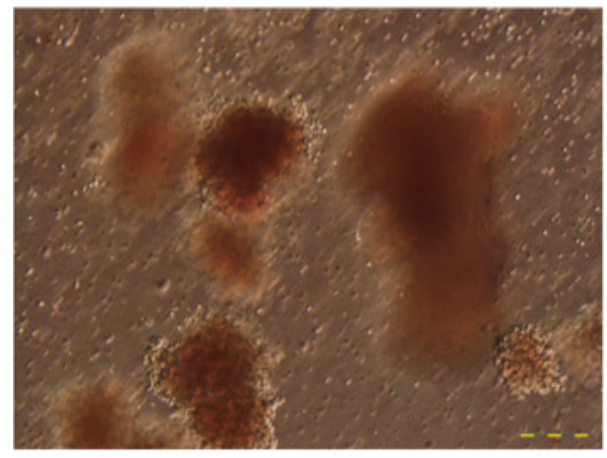

Figure I HSC analysis.

Notes: Samples of MNCs containing HSCs CD34 and CD45 populations were analyzed by flow cytometry. The MNC population was gated in RI based on FSC and SSC (A), and the expression of CD34 (B) and CD34 versus CD45 (C) was analyzed. These HSCs could form CFUs in culture (D) (scale bar I00 $\mu$ m).

Abbreviations: HSCs, hematopoietic stem cells; MNCs, mononuclear cells; FSC, forward scatter; SSC, side scatter; CFUs, colony-forming units; FITC, fluorescein isothiocyanate; APC, antigen-presenting cell.

mice were still alive, while $73.33 \%$ of control mice were still alive.

\section{BC humanized mice}

At day 30 after HSC transplantation, humanized mice were injected with $1 \times 10^{6} \mathrm{BCSC}$ into the fat pad. The results showed that $100 \%$ of humanized mice formed tumors, and invasion appeared at day 60 after BCSC transplantation (Figure 3A and B). More importantly, our results showed that BCSCs invade into the bones of $100 \%$ of the mice (Figure 3C). The results presented in Figure 3D-E clearly showed that the BCSC transplantation also caused mice to die earlier than in the control group.

\section{UCB-derived DCs}

To match HLA between DCs and leukocytes in humanized mice, both DCs and HSCs were isolated from human UCB. MNCs isolated from human UCB were induced by GM-CSF, IL-4, and BCSC-derived antigens for 10 days. The results are presented in Figure 4 and show that the DCs exhibited important characteristics of functional DCs. Specifically, they expressed some costimulator markers, such as CD40, CD80, CD86, and HLA-DR. After phagotogizing with BCSC antigen, they strongly expressed CD40, CD80, CD86, and HLA-DR (Figure 4E-H). More importantly, they successfully stimulated the allogenic T-lymphocytes (Figure 4I) and produced IL-12 (Figure 4J). 

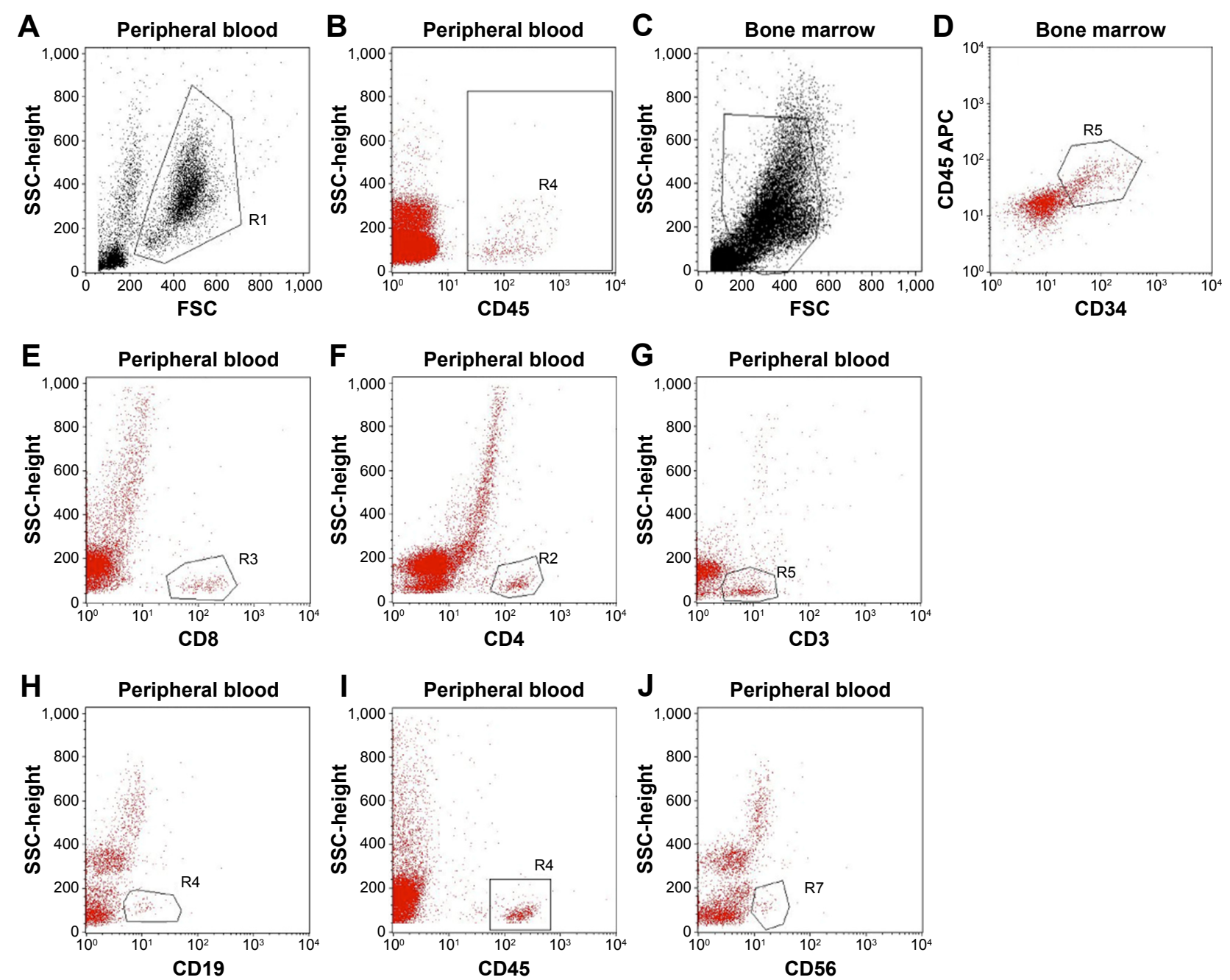

Figure 2 Humanized mice after 28 days of human HSC transplantation.

Notes: Existence of human CD45 leukocytes in murine peripheral blood (A and B). The homing of human HSCs (CD45 ${ }^{+}$CD34 $)$into mouse bone marrow (C and D). Existence of subtype population of human leukocytes in murine blood included CD8 cells (E), CD4 cells (F), CD3 cells (G), CDI9 cells (H), CD45 cells (I), and CD56 cells (J).

Abbreviations: HSCs, hematopoietic stem cells; FSC, forward scatter; SSC, side scatter; APC, antigen-presenting cell.

\section{$D C$ vaccination lengthened the survival of $B C$ humanized mice}

\section{Changes in tumor size}

In our previous study, we found that the tumors in NOD/SCID metastasized after 30 days of treatment. Therefore, in this study, we only monitored the tumor size after treatment with DCs in the first month (30 days) and from day 30 to 75 days of treatment; the efficiency of treatment was evaluated by the survival percentage of mice.

In the first month, tumors clearly responded with DC treatment. Before treatment, the tumor size was about $0.0954 \pm 0.0046 \mathrm{~cm}^{3}$ when injected with $1 \times 10^{6} \mathrm{BCSCs}$ into the fat pad. These tumors strongly increased in size in control mice, while they slowly increased their size in the treated groups. In fact, at day 30 , the tumor size in the control mice increased from $0.044 \pm 0.003 \mathrm{~cm}^{3}$ to $0.745 \pm 0.114 \mathrm{~cm}^{3}$, while in treated mice, the tumor size slightly increased from $0.043 \pm 0.007 \mathrm{~cm}^{3}$ to $0.196 \pm 0.091 \mathrm{~cm}^{3}$. Taken together, we found that the tumor size increased eight times in control mice in the first month, while the tumor size only doubled in treated mice.

\section{Changes in mice body weight in control and treated groups}

The results showed that there was a slight change in the weight of the treated mice compared with that of control mice. Specifically, in control mice, the weight was maintained around $25 \mathrm{~g}$ during the first 30 days of treatment, while in treated mice, the weight of the mice slightly increased in week 1 after vaccinations and the mice maintained the weight during the first month. In the next month, weights of the mice gradually decreased, particularly in mice that eventually died. 

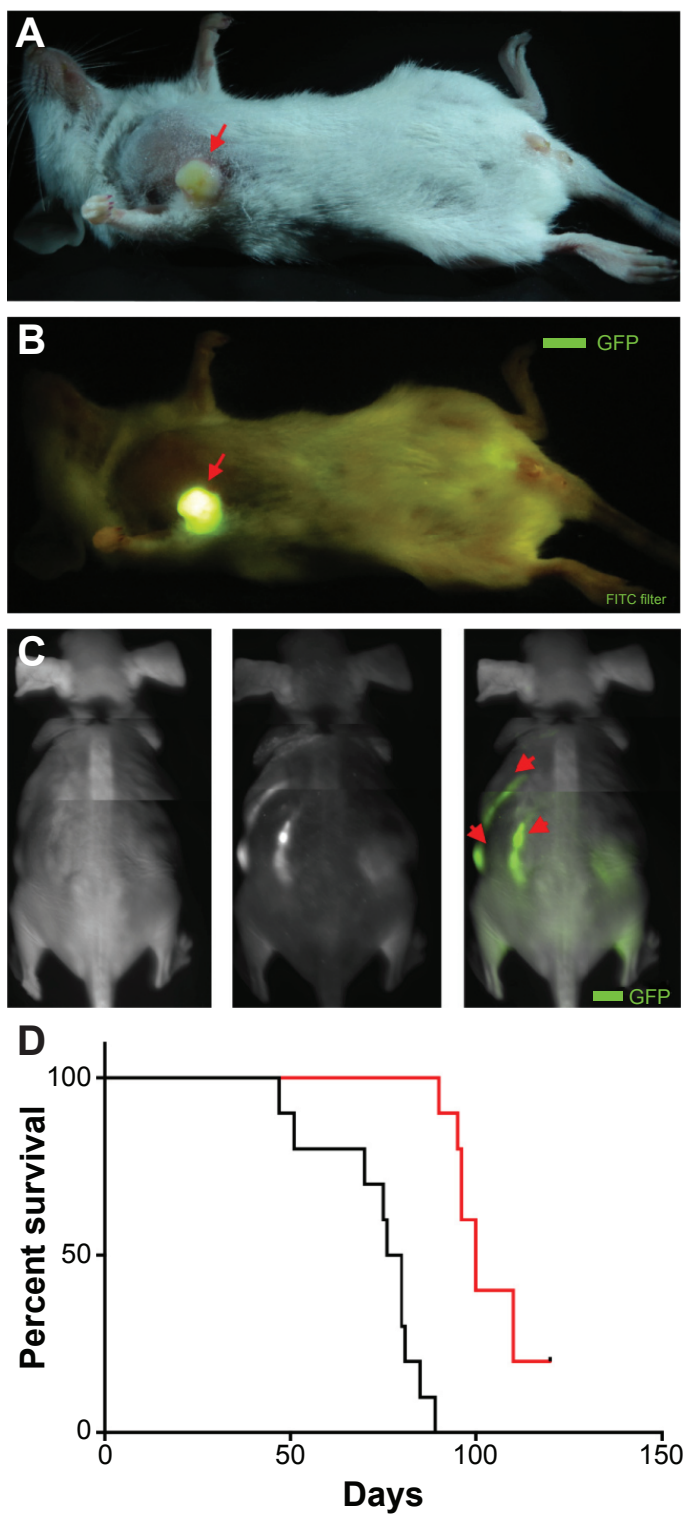

- Humanized mice bearing tumors - Humanized mice

\begin{tabular}{ll}
\hline Log-rank (Mantel-Cox) test & \\
\hline Chi-square & 21.67 \\
$d f$ & 1 \\
$P$-value & $<0.0001$ \\
$P$-value summary & $* * * *$ \\
Are the survival curves sig different? & Yes \\
\hline Gehan-Breslow-Wilcoxon test & \\
\hline Chi-square & 18.09 \\
$d f$ & 1 \\
$P$-value & $<0.0001$ \\
$P$-value summary & $* * * *$ \\
Are the survival curves sig different? & Yes \\
\hline
\end{tabular}

Figure 3 Breast tumor-bearing humanized mice.

Notes: Humanized mouse with breast tumor under white light $(\mathbf{A})$ and under GFP excitation (B). Humanized mouse with metastasis to bone (C). The KaplanMeier test of survival percentage of humanized mice bearing tumors showed that humanized mice bearing tumors had significantly decreased survival percentage compared to humanized mice (D) and were confirmed by Log-rank test and GehanBreslow-Wilcoxon test (E).

Abbreviations: FITC, fluorescein isothiocyanate; sig, significantly; GFPs, green fluorescent proteins.
In almost all cases, the deceased mice weighed between $15 \mathrm{~g}$ and $20 \mathrm{~g}$ in body weight.

\section{BCSC-primed DCs vaccination changed the survival time of treated mice}

All mice were monitored during the 120 days from the first day of the experiment. Figure 5 showed that the BCSCprimed DCs changed the percentage of survival time of treated mice $(P<0.05)$. In fact, after 87 days of experiment, $70 \%$ of treated mice prolonged their survival, while only $40 \%$ of mice survived in the control group. There was $10 \%$ (one of ten mice) of mice in the treated group still alive after 120 days of monitoring, while $100 \%$ of mice in the control group died.

\section{Discussion}

$\mathrm{BC}$ is one of the most common cancers in women and is the second leading cause of death in women. Despite recent advances in the treatment of this disease, the percentage of relapse remains high. In particular, the existing therapies are unable to cure patients with metastases. Therefore, novel therapies are needed to overcome the current paradigm. DC vaccination has been suggested as a suitable strategy for the prevention of relapse in combination with radiation and chemotherapies. Therefore, DC vaccination has been used in the treatment of some diseases, included BC.

However, a recent analysis about $\mathrm{DC}$ vaccination in $\mathrm{BC}$ treatment showed that the efficacy of this therapy is much lower compared with the same in other cancers, such as prostate cancers or melanoma cancer. We proposed that the treatment efficacy depended on antigens used to induce DCs. This study aimed to investigate the treatment efficacy of $\mathrm{BC}$ using $\mathrm{BCSC}$-derived antigen-primed DCs in the $\mathrm{BC}$ humanized mice models.

In the first experiment, the humanized mice models were produced by human HSC transplantation to replace the mouse's blood system. HSCs were collected from human UCB. NOD/SCID mice were transplanted with HSCs according to a previously reported dose. ${ }^{46,47}$ The analyzed results showed that we successfully destroyed the mice bone marrow using busulfan. Although many previous studies used radiation to remove the murine HSCs, a recent publication showed that busulfan was a suitable method to remove the murine HSCs in humanized model production. ${ }^{48-51}$ In this study, we showed that busulfan could efficiently destroy the murine bone marrow. Some previous studies also succeeded to produce humanized models with busulfan as a bone marrow-destroying agent. ${ }^{48,50}$

Although radiation can absolutely kill the murine HSCs, this method can cause a high percentage of death in 

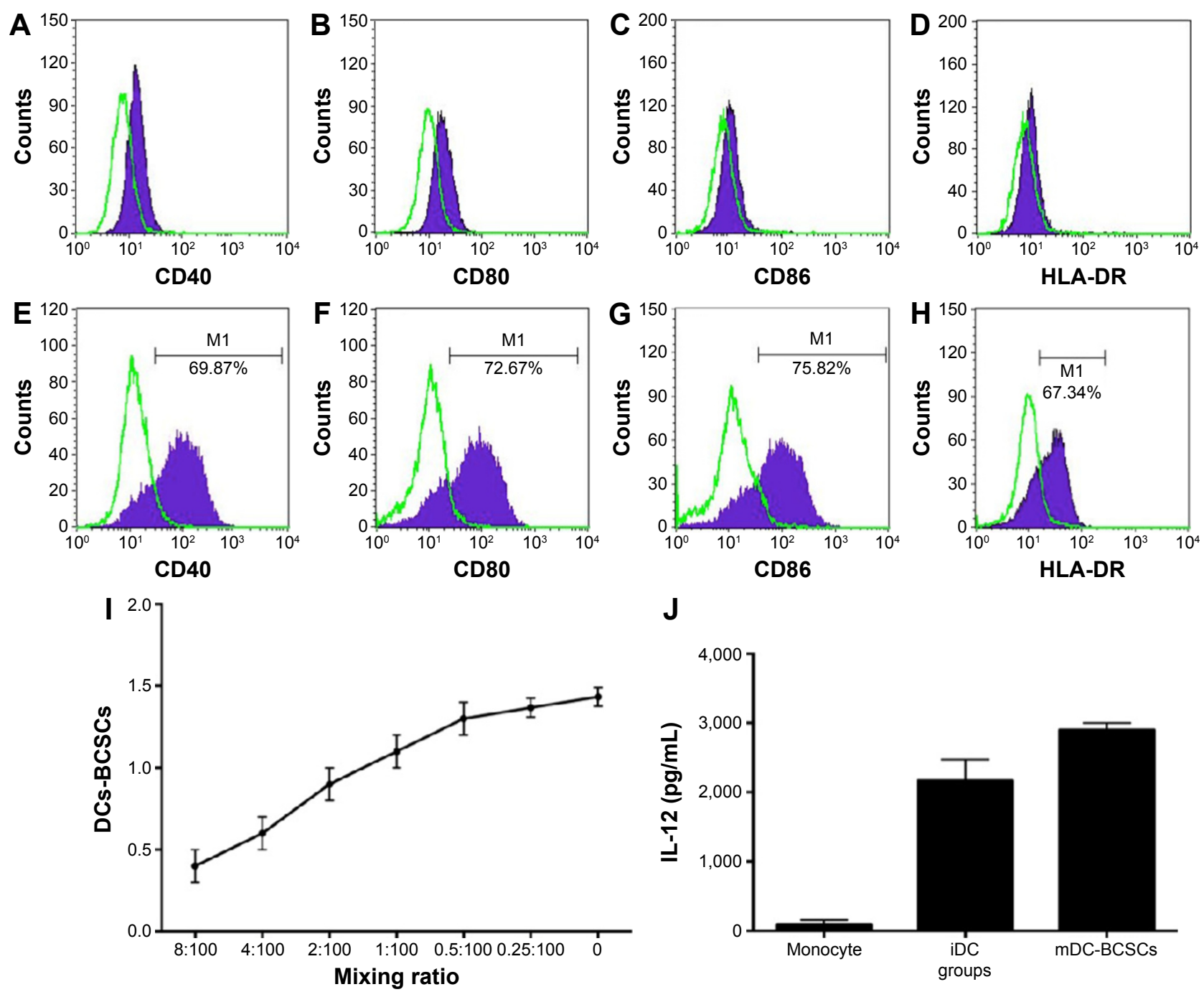

Figure 4 UCB-derived DCs.

Notes: Expression of specific markers of DCs before being induced with GM-CSF and IL-4 (A-D) and after being induced with GM-CSF and IL-4 and BCSC antigen (E-H). MNCs included with GM-CSF and IL-4 and BCSC antigen strongly expressed the DC markers such as CD40, CD80, CD86, and HLA-DR. These cells also successfully stimulated T-lymphocytes (I) and produced IL-I2 (J). Error bars were calculated as standard errors for triplicate.

Abbreviations: UCB, umbilical cord blood; DCs, dendritic cells; GM-CSF, granulocyte macrophage colony-stimulating factor; IL, interleukin; BCSC, breast cancer stem cell; MNCs, mononuclear cells; HLA, human leukocyte antigen; iDC, immature dendritic cell; mDC, mature dendritic cell.

mice. According to Hayakawa et al, ${ }^{48}$ a radiation dose of $91.7 \mathrm{cGy} / \mathrm{min}$ significantly reduced mice survival. Hayakawa et al used busulfan to replace the radiation and showed higher efficacy in humanized mice creation. In our study, at a dose of $25 \mathrm{mg} / \mathrm{kg}$ of busulfan, mice had a significant reduction in body weight, as well as a reduction in WBCs. The results showed that busulfan exhibited its effects on the blood system. In fact, busulfan is an alkylation agent that interferes with the cell division, especially HSCs in murine bone marrow. ${ }^{49,52}$ By flow cytometry analysis, the results also showed that busulfan efficiently reduced the CD4 T-cells compared with CD8 T-cells.

Human HSC transplantation to busulfan-treated NOD/ SCID mice rescued the murine bone marrow. In fact, body weight of the mice increased after mice receiving human HSCs, suggesting that the human HSCs could maintain a healthy hematopoietic system in the mouse. This observation was confirmed by the existence of human CD45 cells in the peripheral blood, and this cell population gradually increased after 2 weeks of HSC transplantation. More importantly, in the WBCs in the peripheral blood, there were $>50 \%$ of cells expressing CD45, which was slightly higher than was reported in a previous study. ${ }^{48}$ According to Hayakawa et al, after receiving $2 \times 10^{6} \mathrm{HSCs}$ for $5-8$ weeks, $\sim 60 \%$ of human CD45 cells were detected in the peripheral blood of the mice.

The gold standard in HSC transplantation is homing of HSC to bone marrow to recover the blood system. The 


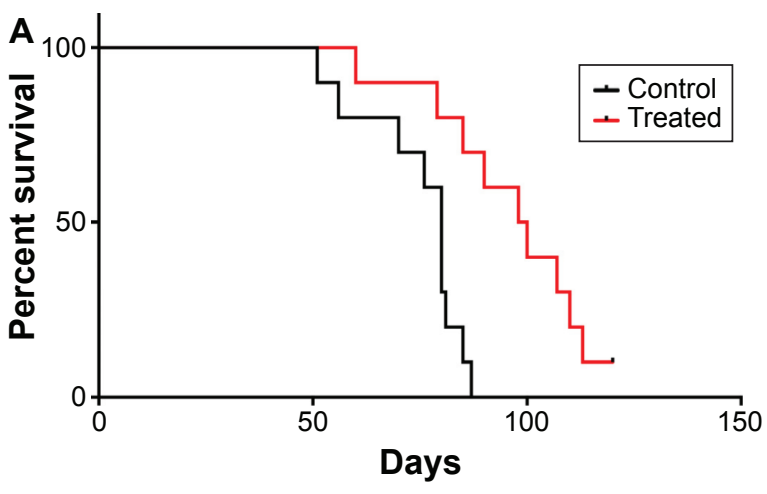

\begin{tabular}{ll}
\hline Log-rank (Mantel-Cox) test & \\
\hline Chi-square & 9.995 \\
$d f$ & 1 \\
$P$-value & 0.0016 \\
$P$-value summary & $* *$ \\
Are the survival curves sig different? & Yes \\
\hline Gehan-Breslow-Wilcoxon test & \\
\hline Chi-square & 7.436 \\
$d f$ & 1 \\
$P$-value & 0.0064 \\
$P$-value summary & $* *$ \\
Are the survival curves sig different? & Yes \\
\hline
\end{tabular}

Figure 5 Treatment efficacy was analyzed by Kaplan-Meier and Log-rank test. KaplanMeier survival percentage of mice in control (black line) and treatment (red line) groups (A); and confirmed by Log-rank test and Gehan-Breslow-Wilcoxon test (B).

Notes: Humanized mice bearing tumors treated with mature DCs had significantly increased survival percentage $P<0.05$. **indicate significant differences.

Abbreviation: DC, dendritic cell.

results showed that $>50 \%$ of $\mathrm{CD} 34^{+}$cells in bone marrow were human $\mathrm{CD}_{3} 4^{+}$cells. The existence of human $\mathrm{CD}^{+} 4^{+}$ cells in bone marrow helped peripheral blood $\mathrm{CD} 45^{+}$cells increase with time. More importantly, human HSCs in the bone marrow successfully differentiated into leukocytes, including T-cells (CD3, CD4, CD8, CD45), B-cells (CD19), and natural killer cells (CD56). In peripheral blood, human IgG was also detected by ELISA, demonstrating that human B-cells were functional. Taken together, these results indicate that we successfully produced humanized mice.

Subsequently, BCSCs with GFP as a reporter were injected into the fat pad to produce the $\mathrm{BC}$ humanized models. In line with the characteristics of BCSCs, the injected cells formed tumors in humanized mice. Based on GFP signal, after 30 days, dead mice revealed that the breast tumors had metastasized into the bone.

The results showed that BCSC-derived antigen-primed DC vaccination significantly inhibited the tumor growth and mice survival. The first evidence of the effects of the DC vaccination was tumor regression, compared with control mice. While the tumor size significantly increased in the control group, tumors in the treated mice maintained or slightly increased in size. The beneficial effect of DC vaccination was also recorded via body weight. In treated mice, their body weight increased. The most important results of BCSC-derived antigen-primed DC vaccination was that the $\mathrm{BC}$ mice survival was significantly prolonged after vaccination.

The main limitation of this study was noncomparison of treatment efficacy between BCSC-derived antigen-primed DCs and BC cell-derived antigen-primed DCs in breast tumor-bearing humanized mice models. However, these results showed that targeting BCSCs exhibited a good effect on inhibiting tumor progression and prolonged survival of breast tumor-bearing humanized mice models.

These results indicated that DCs successfully presented BCSC-derived antigens to other WBCs, such as T-cells and B-cells. These activated WBCs attacked tumor cells or BCSCs and inhibited the tumor progression, as well as tumor invasion. In a recent study, Kai et al used LBH589 and salinomycin to target BCSCs in mice model. ${ }^{38}$ They showed that in xenograft mouse models treated with LBH589 and salinomycin, the drug combination effectively and synergistically inhibited tumor growth of ALDH1positive cells. Using another strategy to target BCSCs, Marcato et al successfully inhibited the BCSCs by oncolytic reovirus. Oncolytic reovirus has the potential to induce tumor regression in BC. ${ }^{53}$ Targeting BCSCs by Ad.mda-7 also showed that Ad.mda-7 inhibited tumor growth associated with a decrease in proliferation and angiogenesis in nude mice models. ${ }^{54}$

\section{Conclusion}

Targeting BCSCs is an important strategy to treat BC. DC therapy is a promising alternative approach to targeting BCSCs. This study showed that DC-primed BCSC-derived antigens efficiently inhibited the tumor progression and prolonged the survival of $\mathrm{BC}$ mice. Moreover, this study also showed that the $\mathrm{BC}$ humanized mice models were feasible models to study and evaluate the DC therapy. Although further studies need to be performed to determine the mechanism and side effects of BCSC-targeting DC-based therapy, these results suggest that BCSC-derived antigen-primed DC therapy is a promising therapy for $\mathrm{BC}$ treatment.

\section{Acknowledgments}

This study was funded by Ministry of Science and Technology under grant number DTDL.2011-T/30.

\section{Author contribution}

PVP performed HSC isolation, developed the humanized NOD/SCID and DC vaccination treatment. HTL and BTV 
carried out humanized mice. VQP and PML prepared the DCs. NLCP and NVT prepared the BC cells and BCSCderived antigens. HTLN and STN analyzed the flow cytometry and monitored the treatment efficacy. TLN and NKP took care of the NOD/SCID mice, performed DC vaccination. All authors contributed toward data analysis, drafting and critically revising the paper and agree to be accountable for all aspects of the work.

\section{Disclosure}

The authors report no conflicts of interest in this work.

\section{References}

1. Dubey AK, Gupta U, Jain S. Breast cancer statistics and prediction methodology: a systematic review and analysis. Asian Pac J Cancer Prev. 2015;16(10):4237-4245.

2. Senda N, Yamaguchi A, Nishimura H, Shiozaki T, Tsuyuki S. Pertuzumab, trastuzumab and docetaxel reduced the recurrence of brain metastasis from breast cancer: a case report. Breast Cancer. 2016;23(2): 323-328.

3. Zekri J, Mokhtar M, Karim SM, et al. Lapatinib-based therapy for women with advanced/metastatic HER2 positive breast cancer. Exp Oncol. 2015;37(2):146-150.

4. Huang L, Chen S, Yang W, et al. Efficacy and safety analysis of trastuzumab and paclitaxel based regimen plus carboplatin or epirubicin as neoadjuvant therapy for clinical stage II-III, HER2-positive breast cancer patients: a phase 2, open-label, multicenter, randomized trial. Oncotarget. 2015;6(21):18683-18692.

5. Cardoso F, Harbeck N, Fallowfield L, Kyriakides S, Senkus E. Locally recurrent or metastatic breast cancer: ESMO clinical practice guidelines for diagnosis, treatment and follow-up. Ann Oncol. 2012;23(suppl 7): vii11-vii19.

6. Turnis ME, Rooney CM. Enhancement of dendritic cells as vaccines for cancer. Immunotherapy. 2010;2(6):847-862.

7. Berzofsky JA, Terabe M, Oh S, et al. Progress on new vaccine strategies for the immunotherapy and prevention of cancer. J Clin Invest. 2004; 113(11):1515-1525.

8. Nencioni A, Grunebach F, Schmidt SM, et al. The use of dendritic cells in cancer immunotherapy. Crit Rev Oncol Hematol. 2008;65(3): 191-199.

9. Ma Y, Shurin GV, Peiyuan Z, Shurin MR. Dendritic cells in the cancer microenvironment. J Cancer. 2013;4(1):36-44.

10. Satthaporn S, Robins A, Vassanasiri W, et al. Dendritic cells are dysfunctional in patients with operable breast cancer. Cancer Immunol Immunother. 2004;53(6):510-518.

11. Banchereau J, Schuler-Thurner B, Palucka AK, Schuler G. Dendritic cells as vectors for therapy. Cell. 2001;106(3):271-274.

12. Xu P, Li PJ, Guo K, et al. Remarkable pathologic change in advanced prostate cancer patient using dendritic cell-cytokine-induced killer combined therapy: a case report. Clin Genitourin Cancer. 2015;13(5): e379-e383.

13. Anguille S, Smits EL, Lion E, van Tendeloo VF, Berneman ZN. Clinical use of dendritic cells for cancer therapy. Lancet Oncol. 2014;15(7): e257-e267.

14. Hoang MD, Jung SH, Lee HJ, et al. Dendritic cell-based cancer immunotherapy against multiple myeloma: from bench to clinic. Chonnam Med J. 2015;51(1):1-7.

15. Pyzer AR, Avigan DE, Rosenblatt J. Clinical trials of dendritic cellbased cancer vaccines in hematologic malignancies. Hum Vaccin Immunother. 2014;10(11):3125-3131.
16. Zheng $\mathrm{K}$, Tan JM, Wu WZ, et al. Adjuvant dendritic cells vaccine combined with cytokine-induced-killer cell therapy after renal cell carcinoma surgery. J BUON. 2015;20(2):505-513.

17. Zhan HL, Gao X, Pu XY, et al. A randomized controlled trial of postoperative tumor lysate-pulsed dendritic cells and cytokine-induced killer cells immunotherapy in patients with localized and locally advanced renal cell carcinoma. Chin Med J (Engl). 2012;125(21):3771-3777.

18. Mayanagi S, Kitago M, Sakurai T, et al. Phase I pilot study of Wilms tumor gene 1 peptide-pulsed dendritic cell vaccination combined with gemcitabine in pancreatic cancer. Cancer Sci. 2015;106(4):397-406.

19. Koido S, Homma S, Okamoto M, et al. Treatment with chemotherapy and dendritic cells pulsed with multiple Wilms' tumor 1 (WT1)-specific MHC class I/II-restricted epitopes for pancreatic cancer. Clin Cancer Res. 2014;20(16):4228-4239.

20. Suehiro Y, Hasegawa A, Iino T, et al. Clinical outcomes of a novel therapeutic vaccine with Tax peptide-pulsed dendritic cells for adult $\mathrm{T}$ cell leukaemia/lymphoma in a pilot study. Br J Haematol. 2015;169(3):356-367.

21. Wilgenhof S, Corthals J, Van Nuffel AM, et al. Long-term clinical outcome of melanoma patients treated with messenger RNA-electroporated dendritic cell therapy following complete resection of metastases. Cancer Immunol Immunother. 2015;64(3):381-388.

22. Lee H, Park HJ, Sohn HJ, Kim JM, Kim SJ. Combinatorial therapy for liver metastatic colon cancer: dendritic cell vaccine and low-dose agonistic anti-4-1BB antibody co-stimulatory signal. J Surg Res. 2011; 169(1):e43-e50.

23. Wu YG, Wu GZ, Wang L, Zhang YY, Li Z, Li DC. Tumor cell lysatepulsed dendritic cells induce a $\mathrm{T}$ cell response against colon cancer in vitro and in vivo. Med Oncol. 2010;27(3):736-742.

24. Wang X, Zhao HY, Zhang FC, Sun Y, Xiong ZY, Jiang XB. Dendritic cell-based vaccine for the treatment of malignant glioma: a systematic review. Cancer Invest. 2014;32(9):451-457.

25. Cho DY, Yang WK, Lee HC, et al. Adjuvant immunotherapy with whole-cell lysate dendritic cells vaccine for glioblastoma multiforme: a phase II clinical trial. World Neurosurg. 2012;77(5-6):736-744.

26. Mao Q, Li L, Zhang C, Sun Y, Liu S, Cui S. Clinical effects of immunotherapy of DC-CIK combined with chemotherapy in treating patients with metastatic breast cancer. Pak J Pharm Sci. 2015;28(3 suppl):1055-1058.

27. Kato Y. [Efficacy of WT1 peptide-/MUC-1 peptide-pulsed dendritic cell therapy in 313 patients with a wide range of cancers]. Gan To Kagaku Ryoho. 2014;41(10):1280-1282.

28. Baek S, Kim CS, Kim SB, et al. Combination therapy of renal cell carcinoma or breast cancer patients with dendritic cell vaccine and IL-2: results from a phase I/II trial. J Transl Med. 2011;9:178.

29. Khattak M, Fisher R, Turajlic S, Larkin J. Targeted therapy and immunotherapy in advanced melanoma: an evolving paradigm. Ther Adv Med Oncol. 2013;5(2):105-118.

30. Simpson EL, Davis S, Thokala P, Breeze PR, Bryden P, Wong R. Sipuleucel-T for the treatment of metastatic hormone-relapsed prostate cancer: a NICE single technology appraisal; an evidence review group perspective. Pharmacoeconomics. 2015;33(11):1187-1194.

31. Brossart P, Wirths S, Stuhler G, Reichardt VL, Kanz L, Brugger W. Induction of cytotoxic T-lymphocyte responses in vivo after vaccinations with peptide-pulsed dendritic cells. Blood. 2000;96(9):3102-3108.

32. Avigan D, Vasir B, Gong J, et al. Fusion cell vaccination of patients with metastatic breast and renal cancer induces immunological and clinical responses. Clin Cancer Res. 2004;10(14):4699-4708.

33. Qi CJ, Ning YL, Han YS, et al. Autologous dendritic cell vaccine for estrogen receptor (ER)/progestin receptor (PR) double-negative breast cancer. Cancer Immunol Immunother. 2012;61(9):1415-1424.

34. Ren J, Di L, Song G, et al. Selections of appropriate regimen of highdose chemotherapy combined with adoptive cellular therapy with dendritic and cytokine-induced killer cells improved progression-free and overall survival in patients with metastatic breast cancer: reargument of such contentious therapeutic preferences. Clin Transl Oncol. 2013; 15(10):780-788. 
35. Al-Hajj M, Wicha MS, Benito-Hernandez A, Morrison SJ, Clarke MF Prospective identification of tumorigenic breast cancer cells. Proc Natl Acad Sci U S A. 2003;100(7):3983-3988.

36. Sharma B, Singh RK. Emerging candidates in breast cancer stem cell maintenance, therapy resistance and relapse. J Carcinog. 2011;10:36.

37. Ojo D, Wei F, Liu Y, et al. Factors promoting tamoxifen resistance in breast cancer via stimulating breast cancer stem cell expansion. Curr Med Chem. 2015;22(19):2360-2374.

38. Kai M, Kanaya N, Wu SV, et al. Targeting breast cancer stem cells in triple-negative breast cancer using a combination of LBH589 and salinomycin. Breast Cancer Res Treat. 2015;151(2):281-294.

39. Diessner J, Bruttel V, Becker K, et al. Targeting breast cancer stem cells with HER2-specific antibodies and natural killer cells. Am J Cancer Res. 2013;3(2):211-220.

40. Pham PV, Phan NL, Nguyen NT, et al. Differentiation of breast cancer stem cells by knockdown of CD44: promising differentiation therapy. J Transl Med. 2011;9:209.

41. Pham PV, Vu BT, Phan NL-C, Le HT, Phan NK. In vitro spontaneous differentiation of human breast cancer stem cells and methods to control this process. Biomed Res Ther. 2015;2(6):7.

42. Phuc PV, Nhung TH, Loan DT, Chung DC, Ngoc PK. Differentiating of banked human umbilical cord blood-derived mesenchymal stem cells into insulin-secreting cells. In Vitro Cell Dev Biol Anim. 2011; 47(1):54-63.

43. Van Pham P, Vu NB, Duong TT, et al. Suppression of human breast tumors in NOD/SCID mice by CD44 shRNA gene therapy combined with doxorubicin treatment. Onco Targets Ther. 2012;5:77-84.

44. Pham PV, Nguyen NT, Nguyen HM, et al. A simple in vitro method for evaluating dendritic cell-based vaccinations. Onco Targets Ther. 2014; 7:1455-1464.

45. Rak J, Mitsuhashi Y, Bayko L, et al. Mutant ras oncogenes upregulate VEGF/VPF expression: implications for induction and inhibition of tumor angiogenesis. Cancer Res. 1995;55(20):4575-4580.
46. Brehm MA, Racki WJ, Leif J, et al. Engraftment of human HSCs in nonirradiated newborn NOD-scid IL2rgamma null mice is enhanced by transgenic expression of membrane-bound human SCF. Blood. 2012 . 119(12):2778-2788.

47. Pearson T, Greiner DL, Shultz LD. Creation of "humanized" mice to study human immunity. Curr Protoc Immunol. 2008;CHAPTER 15 Unit-15.21.

48. Hayakawa J, Hsieh MM, Uchida N, Phang O, Tisdale JF. Busulfan produces efficient human cell engraftment in NOD/LtSz-Scid IL2R $\gamma($ null $)$ mice. Stem Cells. 2009;27(1):175-182.

49. Robert-Richard E, Ged C, Ortet J, et al. Human cell engraftment after busulfan or irradiation conditioning of NOD/SCID mice. Haematologica. 2006;91(10):1384.

50. Singh M, Singh $P$, Gaudray G, et al. An improved protocol for efficient engraftment in NOD/LTSZ-SCIDIL-2Rgammanull mice allows HIV replication and development of anti-HIV immune responses. PLoS One. 2012;7(6):e38491.

51. Hayakawa J, Hsieh M, Phang O, Uchida N, Tisdale JF. Low dose busulfan produces efficient human cell engraftment in immunodeficient mice. Paper presented at: ASH Annual Meeting Abstracts; 2007. Atlanta, Georgia, USA.

52. Brehm MA, Cuthbert A, Yang C, et al. Parameters for establishing humanized mouse models to study human immunity: analysis of human hematopoietic stem cell engraftment in three immunodeficient strains of mice bearing the IL2rgamma(null) mutation. Clin Immunol. 2010; 135(1):84-98.

53. Marcato P, Dean CA, Giacomantonio CA, Lee PW. Oncolytic reovirus effectively targets breast cancer stem cells. Mol Ther. 2009;17(6) 972-979.

54. Bhutia SK, Das SK, Azab B, et al. Targeting breast cancer-initiating/ stem cells with melanoma differentiation-associated gene-7/ interleukin-24. Int J Cancer. 2013;133(11):2726-2736.
OncoTargets and Therapy

\section{Publish your work in this journal}

OncoTargets and Therapy is an international, peer-reviewed, open access journal focusing on the pathological basis of all cancers, potential targets for therapy and treatment protocols employed to improve the management of cancer patients. The journal also focuses on the impact of management programs and new therapeutic agents and protocols on

\section{Dovepress}

patient perspectives such as quality of life, adherence and satisfaction. The manuscript management system is completely online and includes a very quick and fair peer-review system, which is all easy to use. Visit http://www.dovepress.com/testimonials.php to read real quotes from published authors. 